\title{
Nanotechnology and the challenge of clean water
}

\author{
THEMBELA HILLIE ${ }^{1}$ AND MBHUTI HLOPHE ${ }^{2}$ \\ are at the 'National Centre for Nano-Structured Materials, Council for Scientific and Industrial Research, P.0. Box 395, Pretoria 0001, South Africa; ${ }^{2}$ Department of \\ Chemistry, North-West University (Mafikeng campus), Private Bag X2046, Mmabatho 2735, South Africa. \\ e-mail: thillie@csir.co.za; mbhuti.hlophe@nwu.ac.za.
}

\begin{abstract}
Access to 'potable' water would transform the lives of many people in the developing world. Nanotechnology is already being used to remove contaminants from drinking water and increase the availability of fresh water, but there is still a long way to go.
\end{abstract}

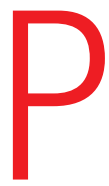

overty and water are closely

linked, and access to water resources has become widely equated with ensuring that basic human needs are met. It is predominantly the poor of the world who depend directly on water and other natural resources for their livelihoods. Water resources must therefore be managed in a sustainable manner in order to maintain the economic, social and environmental functions it provides in contributing to the livelihoods of people.

The complex set of issues involved in the lack of access to potable water and sanitation in various parts of the world have been extensively documented. In 2002, 1.1 billion people did not have access to a reliable water supply and 2.6 billion people - the majority of them living in Africa and Asia - lacked access to adequate sanitation ${ }^{1}$. The consequences of this situation are overwhelming: cholera and other diarrhoeal diseases are major killers, especially for children, in the developing world. Moreover, children often miss school because their schools do not have adequate drinking water and/or sanitation facilities.

Many organizations are considering the potential of nanoscience to solve technical challenges associated with the removal of water contaminants and provide 'potable' water to people in developed and developing countries. A range of water-treatment

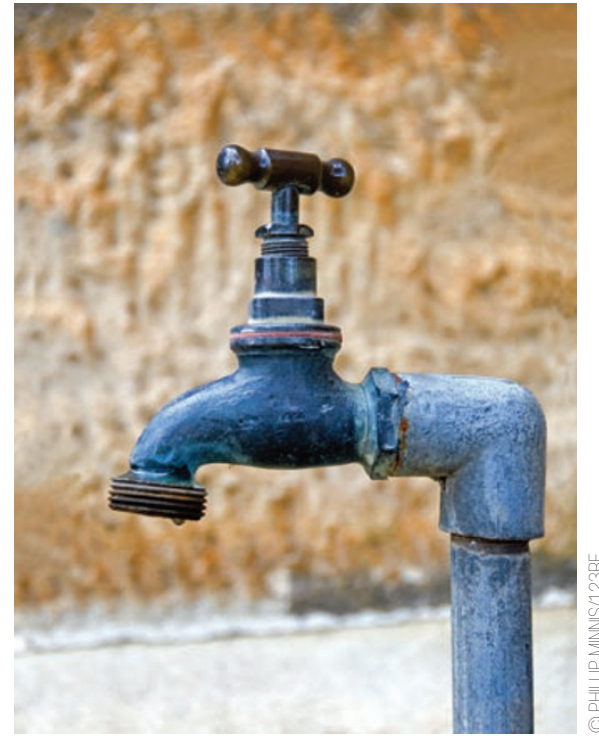

Nanotechnology could increase the availability of clean water in the developing world, but different approaches to technology transfer will be needed for different countries.

devices - including some that incorporate nanotechnology - are already on the market and others are in advanced stages of development.

Conventional water-treatment technologies include filtration, ultraviolet radiation, chemical treatment and desalination, whereas the nano-enabled technologies include a variety of different types of membranes and filters based on carbon nanotubes, nanoporous ceramics, magnetic nanoparticles and other nanomaterials. Ref. 2 contains a comparison between conventional and nano-enabled technologies for water treatment.

Separation membranes with structure at the nanoscale can also be used in lowcost methods to produce potable water. In a recent study in South Africa, several polymeric nanofiltration and reverse osmosis membranes were tested for the treatment of brackish groundwater (water that is salty, but less so than sea water). The tests showed that nanofiltration membranes can produce potable water from the brackish groundwater. As expected, the reverse osmosis membranes removed about $99 \%$ of all the solutes, but the concentrations of essential nutrients, such as calcium and magnesium ions, were reduced to levels that were below the specifications of the World Health Organization standard for drinking water. The product water therefore had to be spiked with these nutrients to provide drinking water of the required quality.

These studies also underline the importance of making communities aware of the actual quality of their drinking water because it is not possible to detect contaminants, for example, by simply observing the physical properties of the water (that is, smell, taste and colour).

However, it is not enough to develop technical solutions to these 
problems - the technology must also be transferred to the country that needs it. In general, direct technology transfer will encounter obstacles and end in failure. To be effective, technology transfer must be accompanied by technology adaptation and technology adoption to take account of the technical capability, infrastructure and market potential of the developing country that needs the technology. Different approaches may well be needed for different developing countries with different sets of socioeconomic realities. Moreover, if technology transfer is not complemented by an effort to develop local capacity, the entire process could prove to be futile. Finally, all stake holders should be involved from the planning stages of a community water-treatment project right through to implementation to enhance transparency and dispel distrust between the parties.

Although much attention has been focused on the potential benefits of water-treatment devices that incorporate nanotechnology, there is also a need for more research to assess the potential human health and environmental risks of nanotechnologies. Although only a few studies have been carried out, some of them indicate that the unique properties of nanomaterials (for example, size, shape, reactivity, conductivity) may cause them to be toxic. There have also been calls for a coordinated risk research agenda to ensure that the right questions are being asked and resources are used efficiently ${ }^{3}$. It is imperative that information about potential risks and risk-management approaches is shared widely, although the risks involved also need to be weighed up against the potential benefits.

Nano-enabled technologies for water treatment are already on the market with nanofiltration currently seeming to be the most mature - and many more are on their way. Although the current generation of nanofilters may be relatively simple, many researchers believe that future generations of water-treatment devices will capitalize on the new properties of nanoscale materials and may prove to be of interest in both developing and developed countries.
Developing countries are at different levels of scientific advancement and have different priorities, so they cannot be subjected to a general prescription for solving water-related and other developmental problems. However, access to potable water is a global problem that requires a concerted effort from all those involved. It needs resources, technology (nano or otherwise) and effective adaptation and adoption strategies that are strongly based on knowledge sharing and local capacity development.

Published online: 21 October 2007.

References

1. Water for Life, Making it Happen (WHO, UNICEF, 2005).

2. Summary of the International Workshop on Nanotechnology, Water and Development (Chennai, India, 10-12 October 2006); available at www.merid.org/nano/waterworkshop 3. Maynard, A. et al. Nature 444, 267-269 (2007).

This article is part of the Global Theme on Poverty and Human Development, organized by the Council of Science Editors. All articles from the Nature Publishing Group are available free at www.nature.com/povhumdev. The content from all participating journals can be found at http://www.councilscienceeditors.org/ globalthemeissue.cfm 